\title{
Erratum to: BMP7 Expression in Esophageal Squamous Cell Carcinoma and Its Potential Role in Modulating Metastasis
}

\author{
Guanghui Xu - Shanhong Tang • Jianjun Yang $\cdot$ Kang Chen · Jianqin Kang • \\ Guohong Zhao $\cdot$ Fan Feng $\cdot$ Xuewen Yang $\cdot$ Lina Zhao $\cdot$ Qun Lu • \\ Li Sun $\cdot$ Liu Hong $\cdot$ Taiqian Gong $\cdot$ Hongwei Zhang
}

Published online: 15 May 2013

(C) Springer Science+Business Media New York 2013

\section{Erratum to: Dig Dis Sci}

DOI 10.1007/s10620-013-2611-3

Some minor data calculating error noticed in Table 1 of the article, correct table is given below:

The online version of the original article can be found under doi:10.1007/s10620-013-2611-3.

G. Xu $\cdot$ S. Tang $\cdot$ J. Yang $\cdot$ K. Chen $\cdot$ J. Kang $\cdot$ G. Zhao

F. Feng $\cdot$ X. Yang $\cdot$ L. Zhao $\cdot$ L. Sun $\cdot$ L. Hong $\cdot$ H. Zhang $(\bowtie)$

State Key Laboratory of Cancer Biology, Xijing Hospital of

Digestive Diseases, The Fourth Military Medical University,

15 Changle Western Road, Xi'an 710032,

People's Republic of China

e-mail: zhanghw41@126.com; zhanghw@fmmu.edu.cn

S. Tang

Department of Digestion, General Hospital of Chengdu Military

Command, Chengdu 610083, Sichuan Province, China

Q. $\mathrm{Lu}$

Department of Operative Dentistry and Endodontic, College

of Stomatology, Fourth Military Medical University,

Xi' an 710032, People's Republic of China

T. Gong $(\bowtie)$

Department of Thoracic Surgery, Daping Hospital,

Third Military Medical University, Chongqing 400042,

People's Republic of China

e-mail: gongtq@yahoo.com.cn 
Table 1 Relationship between BMP7 expression and clinical features of ESCC BMP expression

ESCC Esophageal squamous cell carcinomas, $S D$ standard deviation, $U$ cervical upper thoracic esophagus, $M$ middle esophagus, $L$ lower thoracic and abdominal esophagus, AJCC

American Joint Committee on Cancer

\begin{tabular}{|c|c|c|c|c|}
\hline \multirow[t]{2}{*}{ Parameter } & \multicolumn{3}{|c|}{ BMP Expression } & \multirow[t]{2}{*}{$p$ Value } \\
\hline & - & + & ++ & \\
\hline Age $($ mean $\pm \mathrm{SD})$ & $58.39 \pm 7.93$ & $58.91 \pm 7.35$ & $58.58 \pm 7.76$ & \\
\hline Sex & & & & 0.54 \\
\hline Male & 42 & 28 & 33 & \\
\hline Female & 12 & 7 & 5 & \\
\hline Size $(\mathrm{cm})$ & & & & 0.07 \\
\hline$\leq 2$ & 13 & 5 & 4 & \\
\hline $2-5$ & 33 & 21 & 19 & \\
\hline$\geq 5$ & 8 & 9 & 15 & \\
\hline Histological cell type & & & & 0.11 \\
\hline Well & 27 & 19 & 10 & \\
\hline Moderate & 18 & 10 & 20 & \\
\hline Poor & 9 & 6 & 8 & \\
\hline Location & & & & 0.50 \\
\hline $\mathrm{U}$ & 12 & 5 & 5 & \\
\hline M & 28 & 18 & 17 & \\
\hline $\mathrm{L}$ & 14 & 12 & 16 & \\
\hline AJCC stage & & & & $<0.001$ \\
\hline IA, IB & 11 & 5 & 4 & \\
\hline IIA, IIB & 37 & 18 & 13 & \\
\hline IIIA, IIIB, IIIC & 6 & 12 & 21 & \\
\hline pT staging & & & & 0.14 \\
\hline $\mathrm{T} 1$ & 7 & 4 & 1 & \\
\hline $\mathrm{T} 2$ & 27 & 17 & 14 & \\
\hline $\mathrm{T} 3+\mathrm{T} 4$ & 20 & 14 & 23 & \\
\hline LN metastasis & & & & $<0.001$ \\
\hline Negative (N0) & 33 & 13 & 6 & \\
\hline Weak positive (N1) & 12 & 17 & 14 & \\
\hline Strong positive (N2-N3) & 9 & 5 & 18 & \\
\hline
\end{tabular}

